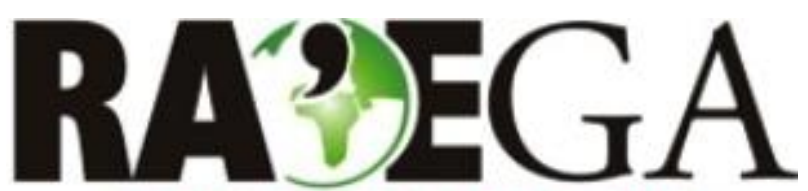

O ESPAÇO GEOGRÁFICO EM ANÁLISE

\title{
DISTRIBUIÇÃO GEOGRÁFICA DAS COLETAS, ESTUDOS E PUBLICAÇõES ACADÊMICAS SOBRE NOVE PLANTAS MEDICINAIS UTILIZADAS PARA O CONTROLE DE DIABETES EM MATO GROSSO
}

\section{GEOGRAPHICAL DISTRIBUTION OF COLLECTIONS, ACADEMIC STUDIES AND PUBLICATIONS ON NINE PLANTS MEDICAL USED FOR DIABETES CONTROL IN MATO GROSSO}

\author{
Isabela Silva Matos ${ }^{1}$, Arno Rieder ${ }^{2}$
}

\section{RESUMO}

Diabetes mellitus (DM) é uma síndrome metabólica que afeta parte da população. Plantas medicinais são formas alternativas terápicas no seu controle. Resultados de estudos com plantas medicinais geralmente estão dispersos espacialmente. Objetivou-se mostrar a distribuição geográfica de coletas, estudos e publicações de 9 plantas medicinais utilizadas no controle de diabetes em Mato Grosso. As fontes se deram através do Google Acadêmico, aplicando 4 modalidades de busca (M3, M4, M5 e M6) e 6 variantes. Aproveitou-se 136 textos cujos dados foram lançados em um banco eletrônico. Os continentes que mais contribuíram com coletas foram: Americano (31,61\%), Asiático (30,14\%) e Africano (23,52\%) e países como Brasil (26,49\%), Índia e Nigéria com 29 coletas cada (24,78\%). Os continentes e países dos locais de estudos seguem a mesma ordem dos de coleta, respectivamente: $(36,28 \%)$, $(35,39 \%)$ e $(27,43 \%) ;(25,66 \%),(28=24,77 \%$ x 2). As publicações frequentemente estavam na América $(46,46 \%)$, Ásia $(28,28 \%)$ e Europa $(13,13 \%)$ e em países como Brasil $(25,25 \%)$, Índia $(23,23 \%)$ e Estados Unidos $(10,10 \%)$. Conclui-se que, os locais de coleta distribuem-se diferentemente entre 4 continentes, 18 países e entre vários estados e cidades, assim como os estudos, com locais de sua realização. As publicações migram dos países de estudo para países desenvolvidos.

PALAVRAS-CHAVE: Espécies; Síndrome Metabólica; Geografia.

\section{ABSTRACT}

Diabetes mellitus (DM) is a metabolic syndrome that affects part of the population. Medicinal Plants are alternative ways terápicas in your control. Results of studies with medicinal plants are generally dispersed spatially. We aimed to show the geographic distribution of collections, studies and publications of 9 medicinal plants used in the control of diabetes in the state of Mato Grosso. The sources came through Google Scholar, using 4 methods of search (M3, M4, M5 and M6) and 6 variants. It took 136 texts whose data were released in an electronic database. The continents that contributed most with collections were: American (31.61\%), Asia (30.14\%) and Africa (23.52\%) and countries such as Brazil (26.49\%), India and Nigeria with 29 collections each (24.78\%). Continents and studies of local countries follow the same order of collection, respectively: (36.28\%) (35.39\%) and $(27.43 \%) ;(25.66 \%),(28 \times 2=$ 24.77\%). The publications were often in America (46.46\%), Asia (28.28\%) and Europe (13.13\%) and in countries such as Brazil $(25.25 \%)$, India (23.23\%) and the United States (10.10\%). In conclusion, the sampling sites are distributed differently across 4 continents, 18 countries and across several states and cities, as well as studies, with local realization. The publications migrate more the study countries to developed countries.

KEY-WORDS: Species; Metabolic Syndrome; Geography.

Recebido em: 16/02/2016

Aceito em: 22/06/2017

\footnotetext{
${ }^{1}$ Universidade do Estado de Mato Grosso, Cáceres/MT e-mail: isa-bela31@hotmail.com

${ }^{2}$ Universidade do Estado de Mato Grosso, Cáceres/MT, e-mail: riederarno@gmail.com
} 


\section{DISTRIBUIÇÃO GEOGRÁFICA DAS COLETAS, ESTUDOS E PUBLICAÇÕES ACADÊMICAS SOBRE NOVE PLANTAS MEDICINAIS UTILIZADAS PARA O CONTROLE DE DIABETES EM MATO GROSSO}

\section{INTRODUÇÃO}

\section{APRESENTAÇÃO}

Trata-se de um estudo sobre a distribuição geográfica de produção e disponibilização de conhecimentos na temática "plantas medicinais para controle de diabetes", contemplando 9 espécies utilizadas em Mato Grosso.

\section{CONSIDERAÇÕES INICIAIS}

Para entender e poder estimular melhor o desenvolvimento local, regional e global é essencial saber como o acesso a informação, sua disponibilidade e o domínio de conhecimentos gerais e específicos estão distribuídos nestes lugares. A distribuição geopolítica de instituições de pesquisa e de publicação de resultados são indicadores importantes para inferir sobre o desenvolvimento de determinada área de conhecimento de povos. O ritmo de desenvolvimento de um povo está relacionado às políticas de seus líderes e governos, principalmente as condizentes a educação, cultura, ciência e tecnologia. Os que mais bem investem priorizando estes setores estarão melhor situados e em direção à dianteira do desenvolvimento.

Objetos deste trabalho, plantas com propriedades medicinais, geograficamente, estão distribuídas pelo mundo todo. As conhecidas e ainda a elucidar se constituem em um importante patrimônio para a humanidade. Plantas para auxiliar no controle do diabetes, constituem-se promissoras alternativas terapêuticas. Muitas já são usadas popularmente. Mas desafiam a ciência e a tecnologia, seja no aprimoramento de informações sobre segurança e eficácia como na busca do novo, ampliado e aprofundado conhecimento e; também para o desenvolvimento de produtos terapêuticos.

\section{DISTRIBUIÇÃO ESPACIAL DE PESQUISAS: SITUAÇÃO E POSSIBILIDADES}

Nesta temática, as instituições de pesquisa (IP), pesquisadores em ação (PA) e as publicações dos resultados (PR) de seus projetos, continuam a aumentar pelo mundo. Isto é motivado por razões diversas (políticas, sociais, econômicas, culturais, etc.). A ocorrência disto (IP, PA, PR) espacialmente é assimetricamente distribuída entre continentes e países. Ao desejar-se abordar a distribuição espacial (IP, PA, $P R$ ) para focos mais específicos, como de plantas medicinais para controle do diabetes e complicações associadas, as informações ainda precisam ser mais bem organizadas e categorizadas. Ao sintetizá-las é facilitada sua retratação, análise, interpretação e compreensão de significados. Estas possibilidades estarão alimentando e subsidiando melhor a revisão, ajustes e implantação de ações à otimização de políticas de pesquisas aos governos.

\section{CARACTERIZAÇÃO E OCORRÊNCIA DO DIABETES}

O diabetes mellitus (DM) caracteriza-se por um conjunto de doenças metabólicas que apresenta hiperglicemia, consequente de defeitos de secreção e/ou de ação da insulina. (BRASIL, 2006, p.9).

Segundo a Organização Mundial de Saúde (OMS), a incidência e prevalência de diabéticos crescem principalmente nos países em desenvolvimento e mais acentuadamente para o grupo que ultrapassa 45 anos de idade e; em todo mundo com prováveis 177 milhões de diabéticos em 2000 e, 350 milhões no ano 2025 (BRASIL, 2006, p.9). No Brasil, os casos de diabetes também estão aumentando e em maior proporção em pessoas com mais de 60 anos (SBD, 2013-2014, p.1). Este perfil dinâmico do diabetes pode estar estimulando diferentemente povos, nações que habitam os vários continentes, no estudo de alternativas à terapia desta síndrome metabólica.

\section{FITOTERAPIA NO CASO DE DIABETES}

Muitas pessoas preferem as terapias alternativas aos tratamentos convencionais (REZENDE, COCCO, 2002, p.283) e optam por plantas medicinais. 


\section{DISTRIBUIÇÃO GEOGRÁFICA DAS COLETAS, ESTUDOS E PUBLICAÇÕES ACADÊMICAS SOBRE NOVE PLANTAS MEDICINAIS UTILIZADAS PARA O CONTROLE DE DIABETES EM MATO GROSSO}

Conforme a Resolução n. 26 da Diretoria Colegiada (RDC) da Agência Nacional de Vigilância Sanitária (ANVISA), publicada em 13 de maio de 2014, planta medicinal é: “espécie vegetal, cultivada ou não, utilizada com propósitos terapêuticos" (BRASIL, 2014, p.4). A RDC no 26/2014 (BRASIL, 2014) estabelece clareza conceitual, norteia ações e expressa um grande avanço na valorização dos recursos fitoterápicos e na validação de seu uso em todo país.

As plantas medicinais são fontes de novos fármacos e fitoterápicos (BRASIL, 2012, p.13). O conhecimento popular sobre plantas medicinais, transmitido entre pessoas e gerações pode ser o único recurso terapêutico de certas pessoas e etnias (MACIEL et al., 2002, p.429).

\section{USO REGIONAL DE PLANTAS MEDICINAIS}

No Brasil plantas medicinais são mais usadas na região norte (MEDEIROS, 2014, p.18) e, no Estado de Mato Grosso, vários estudos também mencionam este uso (RIEDER, GUARIM NETO, 2012, p.23). Há diversas plantas medicinais benéficas para diabéticos. Segundo Negri (2005, p.122) 82 espécies são aplicadas pela medicina tradicional chinesa; 37 na terapia do diabetes no sudoeste do Marrocos.

\section{INVESTIMENTOS EM CIÊNCIA E TECNOLOGIA E SEUS REFLEXOS.}

Embora haja menções em Volpato et al. (2002, p.36) de sugestões que plantas medicinais possam substituir terapias convencionais hipoglicemiantes, estudos com fito-componentes ativos precisam ser aprofundados e ampliados. Pois o conhecimento existente é variado, incipiente e em alguns casos com resultados contraditórios. Os principais desafios são comprovar eficácia e segurança das espécies de interesse dos mais diversos lugares do mundo.

Segundo Contini e Séchet (2011, p.31) a situação da ciência e tecnologia de um país é um reflexo dos investimentos gerais, no capital humano e na produção científica. Esta análise posiciona $o$ país no cenário científico e tecnológico mundial e, permite subsidiar políticas ao replanejamento de ações e fortalecimento dos entes melhorarem resultados.

Conforme Guimarães (2004, p.304) o complexo educacional universitário e, consequentemente, o sistema de ciência e tecnologia foram estruturados tardiamente no Brasil e ainda não consolidados.

Para apoio à pesquisa em saúde no Brasil, entre 2004 e o final de 2006, a Secretaria de Ciência, Tecnologia e Insumos Estratégicos (MS/SCTIE/Decit) teria investido cerca de US\$100 milhões (GUIMARÃES, 2006, p.8). Levantamento realizado pelo Forum Global de pesquisa em Saúde, mostra que em 2001 foram despendidos quase US\$106 bilhões com pesquisa e desenvolvimento em saúde no mundo.

MERCADO, ESTUDOS E PUBLICAÇÕES SOBRE PLANTAS MEDICIANAIS: ESPAÇO E TEMPO

O mercado de produtos de plantas medicinais se desenvolveu vertiginosamente nos últimos 10 anos do século passado, principalmente em países da Europa como Alemanha, França, Itália, Reino Unido, Espanha e mais recentemente nos Estados Unidos da América (CALIXTO, 2000, p.180).

Menções de Badke (2008, p.22) indicam que estudos sobre poder terapêutico de plantas teriam iniciado em 1906, sem citar onde. Conforme Motta et al. (2009, p.37), as pesquisas qualitativas sobre o uso de medicina alternativa e complementar no tratamento de pacientes com DM tipo 2, realizam-se especialmente em países desenvolvidos e; sobre o uso da medicina tradicional mais em países pobres ou em desenvolvimento.

No mundo, muitas plantas medicinais são estudadas para avaliar propriedades hipoglicemiantes (PEREIRA, 2012, p.3).

Contudo, entre muitos resultados de estudos com plantas medicinais, considerável quantidade está em acessos restritos ou não disponíveis publicamente e, ainda dispersos espacialmente e; sua origem, hospedagem (bibliotecas: bases físicas e virtuais) e domínio predominam em alguns países. 


\section{DISTRIBUIÇÃO GEOGRÁFICA DAS COLETAS, ESTUDOS E PUBLICAÇÕES ACADÊMICAS SOBRE NOVE PLANTAS MEDICINAIS UTILIZADAS PARA O CONTROLE DE DIABETES EM MATO GROSSO}

\section{OBJETIVOS}

O objetivo deste trabalho é mostrar a distribuição geográfica das coletas, estudos e publicações acadêmicas, sobre 9 plantas medicinais aplicadas para o controle de diabetes utilizadas em Mato Grosso, Brasil.

\section{MATERIAIS E MÉTODOS}

As 9 plantas abordadas neste estudo são de um pacote (conjunto de 9-11 espécies de plantas), de 13 distribuídas entre pesquisadores do grupo FLOBIO (Estudo da Flora Bioativa), originados da lista constante no livro de Rieder e Guarim Neto (2012, p.54), a qual trata de plantas medicinais utilizadas para o controle de diabetes em Mato Grosso (MT), Brasil (BR).

As famílias e espécies dessas são: a) Caricaceae: Carica papaya Linn. b) Salicaceae: Casearia sylvestris Sw. c) Euphorbiaceae: Croton goyazensis Müll. Arg., Croton salutaris Casar., Croton urucurana Baill., Jatropha elliptica (Pohl) Oken., Jatropha urens L., Phyllanthus niruri Linn. e Phyllanthus tenellus Roxb.

Para alimentar e responder aos objetivos, foram reunidos dados colhidos em publicações, acessadas na internet via online, através do Google Acadêmico (GA) aplicando-se como procedimento para recuperação de dados, 4 modalidades (M) de busca conforme Rieder e Rodrigues (2012, p.7): M3 (no título: "nome científico" "diabetes"), M4 (no título: "nome científico" "diabetes" "abstract"), M5 (tudo no título: "nome científico" "diabetes"), M6 ("nome científico" "diabetes" "abstract") com 6 variantes cada modalidade ("diabetes", "diabetic", "antidiabetic", "hypoglycemic", "hyperglycemic" e "antihyperglycemic"). As palavras-chave foram inseridas na caixa de pesquisa e dado "enter" ocasionando a localização e recuperação de dados mostrados na tela do computador para leitura e seleção.
São considerados textos aproveitados, aqueles que relacionam a espécie visada com atividade antidiabética ou alguma complicação associada. Os aproveitados foram categorizados em 2 tipos de vínculo: direto (quando o artigo aborda diretamente, em seu estudo, a planta para o diabetes) e indireto (trabalho que não pesquisa diretamente a planta para o diabetes, mas faz referência proveitosa para este fim).

As buscas na internet foram realizadas a partir de Cáceres (MT, BR) do período de 11 jul 2013 à 30 jan 2014. Os textos recuperados foram lidos para triagem; os condizentes com os objetivos foram selecionados; os dados foram coletados e lançados em um banco de dados eletrônico (Microsoft Excel 2010), estruturado para contemplar e alimentar análises pertinentes. As análises se utilizaram de ferramentas (proporcionalidade, Teste $\chi^{2}$, significância das diferenças) e programas apropriados de estatística (Excel) e outros (SPSS).

\section{RESULTADOS E DISCUSSÃO TEXTOS APROVEITADOS}

Conforme a metodologia empregada neste trabalho, se recuperaram centenas de textos, dos quais se aproveitou 136 (Anexo 1 Site acesso). Das 9 espécies de plantas, apenas 7 apresentaram estudos aproveitados: Carica papaya (68), Casearia sylvestris (7), Croton goyazensis (1), Croton urucurana (4), Jatropha elliptica (1), Phyllanthus niruri (49) e Phyllanthus tenellus (6). Para 2 espécies não foram recuperados textos aproveitáveis, sendo essas: Croton salutaris e Jatropha urens.

\section{LOCAIS DE COLETA}

As distribuições geográficas dos locais de coleta do material estudado, revelado nas publicações aproveitadas, estão distribuídas por diversos lugares do mundo (Figura 1) 


\section{DISTRIBUIÇÃO GEOGRÁFICA DAS COLETAS, ESTUDOS E PUBLICAÇÕES ACADÊMICAS SOBRE NOVE PLANTAS MEDICINAIS UTILIZADAS PARA O CONTROLE DE DIABETES EM MATO GROSSO}
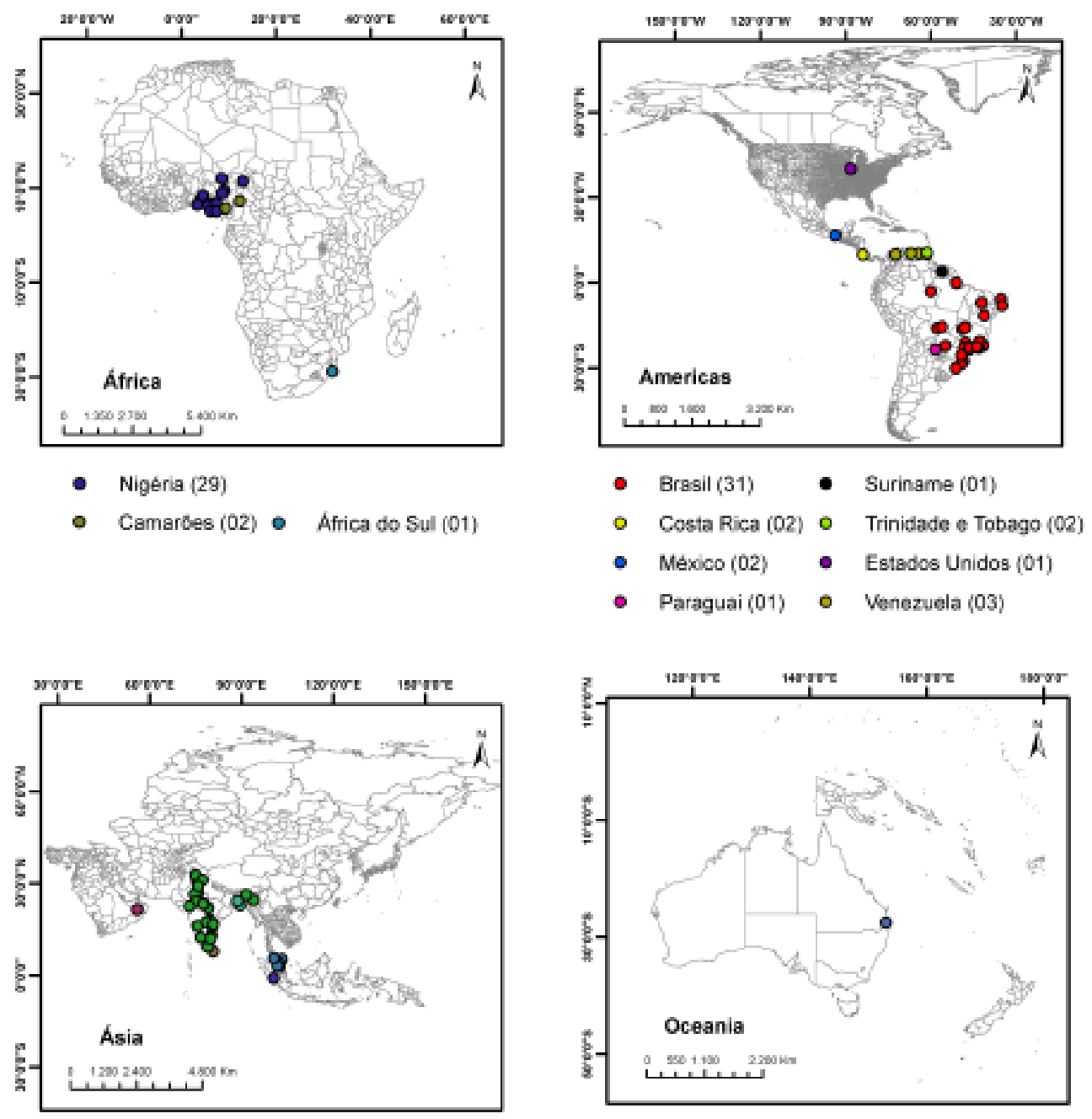

- Bangladesh (02) - Malásia (07)

- İndia (29) - Oma (01)

- Indonésia (01) - Sri Lanka (01)

- Austrália (01)

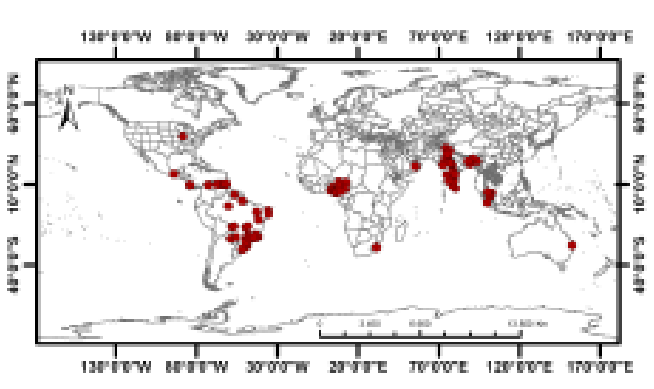

\begin{tabular}{|c|}
\hline $\begin{array}{l}\text { Materiais coletados para os estudos } \\
\text { dos textos aproveitados que se referem a } \\
\text { fitoterapia no controle de diabetes }\end{array}$ \\
\hline - Locais de Coleta 5 Divisăo Pólitica \\
\hline 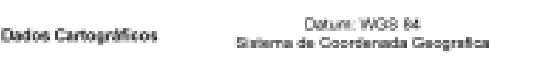 \\
\hline $\begin{array}{l}\text { Thxes E Uins } \\
\text { Jatrozole }\end{array}$ \\
\hline
\end{tabular}

Figura 01 - Distribuição geográfica dos materiais coletados para os estudos referentes aos textos aproveitados sobre fitoterapia no controle de diabetes envolvendo sete espécies utilizadas em Mato Grosso (MT), BRASIL (BR). Fonte de dados: Recuperado na internet via Google acadêmico. Acessado em: Cáceres, MT, BR, entre 11 jul. 2013 à 30 jan. 2014. Organização: os autores. 


\section{DISTRIBUIÇÃO GEOGRÁFICA DAS COLETAS, ESTUDOS E PUBLICAÇõES ACADÊMICAS SOBRE NOVE PLANTAS MEDICINAIS UTILIZADAS PARA O CONTROLE DE DIABETES EM MATO GROSSO}

As 2 espécies que mais contribuíram para o total de coletas foram Carica papaya (50\%) e Phyllanthus niruri (36,02\%) (Figura 2), ambas bem adaptadas ao ambiente tropical. Conforme Da Silva et al. (2013a, p.797) a C. papaya é uma fruteira comum em quase todos os países da américa tropical, considerada como uma das mais cultivadas e consumidas nas regiões tropicais e subtropicais do mundo.

Os continentes com maiores contribuições para o total de coletas foram: o americano $(43=31,61 \%)$, seguido do asiático $(41=30,14 \%)$ e africano $(32=23,52 \%)$, os quais, pelo menos em parte, estão em faixa tropical. No continente americano, a América do Sul se destacou (36=26,47\%). Não se identificou o continente de material de coleta em 19 estudos.

Os países identificados (117) onde ocorreram mais coletas foram Brasil (31=26,49\%), seguido pela Índia e Nigéria com 29 coletas de cada uma $(24,78 \%)$. Esses 3 países também contêm ambientes tropicais, pelo menos em parte deles. Há uma grande diversidade de estados (63) e cidades (75) em que foram realizadas as coletas (Figura 1 ).

As proporções de textos aproveitados, para local de coleta das espécies, variaram entre os continentes ( $\chi 2=38,47 ; \mathrm{GL}=6 ; \alpha=9,0537 \mathrm{E}-07$ ). As diferenças maiores entre frequências observadas (Fo) e esperadas (Fe) são constatadas na espécie $C$. papaya, nos continentes africano e americano. Diante da hipótese da nulidade, esperava-se 16 textos com coletas no continente africano, mas ocorreram 24 enquanto, no continente americano esperava-se 22 e ocorreram apenas 10. Para P. niruri, as diferenças entre frequências esperadas e observadas não são tão expressivas.

Para as demais espécies, esperavam-se 7 coletas no continente americano e ocorreram 17 enquanto no asiático esperava-se 6 e verificou-se 1 apenas. Isto revela que os estudos com a $C$. papaya coletaram mais a planta no continente africano e menos no americano, em relação ao esperado. As coletas das 7 espécies pesquisadas se deram em vários países (Figura 2).

As coletas "identificadas" para as 7 espécies estão distribuídas entre 18 países, mas as coletas de $C$. papaya e $P$. niruri ocorreram em 13 e 10 países respectivamente. Enquanto as "demais espécies" concentraram suas coletas no Brasil, exceto J. elliptica. 


\section{DISTRIBUIÇÃO GEOGRÁFICA DAS COLETAS, ESTUDOS E PUBLICAÇÕES ACADÊMICAS SOBRE NOVE PLANTAS MEDICINAIS UTILIZADAS PARA O CONTROLE DE DIABETES EM MATO GROSSO}

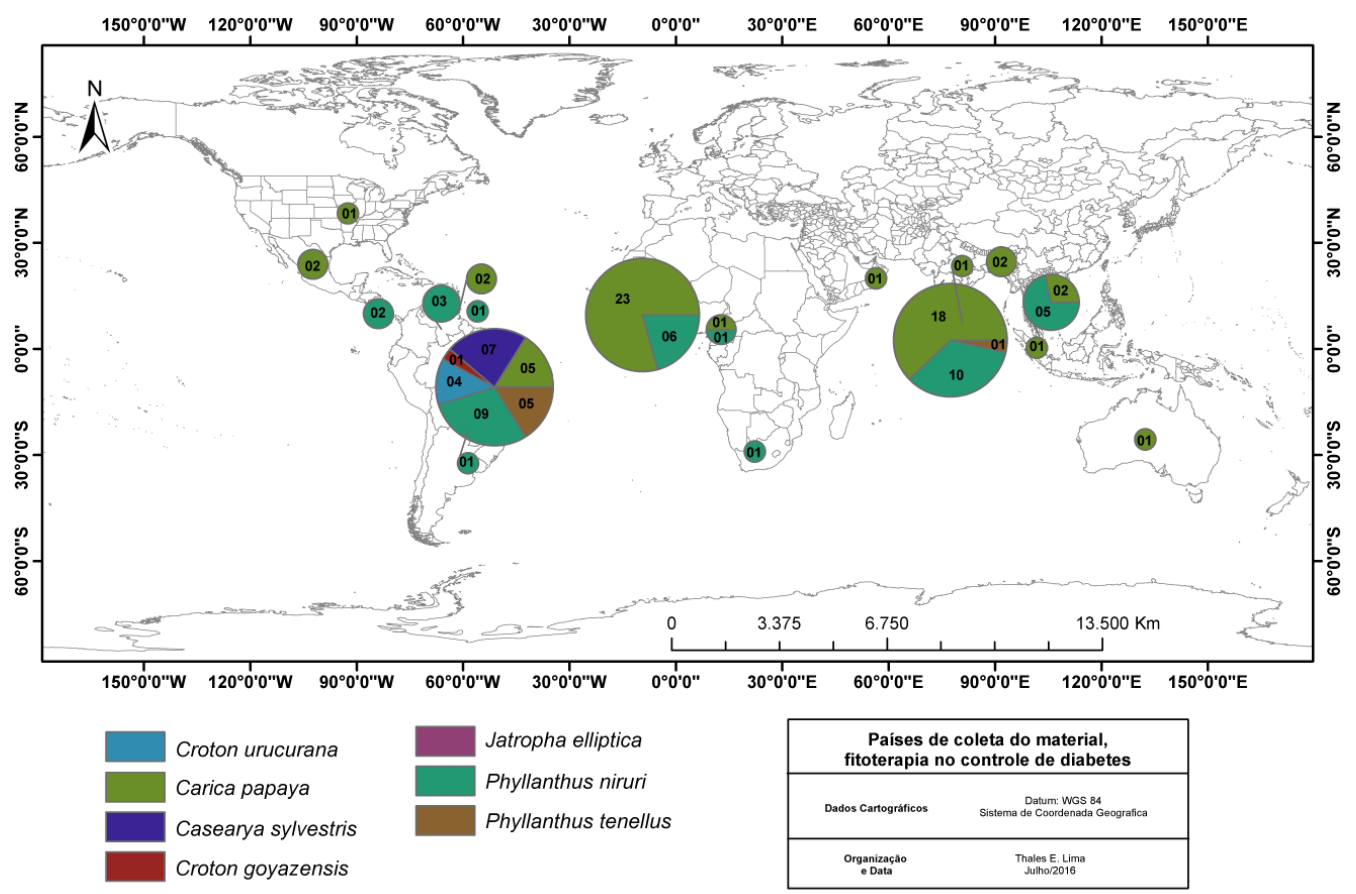

Figura 02 - Espécies e lugar geopolítico de coleta do material usado nos estudos dos textos aproveitados que se referem a fitoterapia ao controle de diabetes envolvendo sete espécies utilizadas em Mato Grosso (MT), BRASIL (BR).Fonte de dados: Recuperado na Internet via Google acadêmico. Acessado em: Cáceres, MT, BR, entre 11 jul. 2013 à 30 jan. 2014. Organização: os autores.

As coletas, com países "identificados", para C. papaya (60) foram mais frequentes na Nigéria $(23=38,33 \%)$ e Índia (18=30\%); para $P$. niruri (39) foram Índia $(10=25,64 \%)$ e Brasil $(9=23,07 \%)$.

Conforme Dantas et al. (1999, p.1) os principais países produtores de mamão no mundo, em 1997, eram Nigéria (23,57\%), Índia $(17,67 \%)$ e Brasil $(11,39 \%)$. Este fato pode estar associado com os locais mais frequentes de coleta dessa espécie para as pesquisas, revelando coerência com o presente estudo quanto a distribuição das coletas nos países identificados.

$\mathrm{Na}$ Índia houve coleta de 3 espécies: $\mathrm{C}$. papaya, P. niruri e P. tenellus. Em Camarões,
Malásia e Nigéria ocorreram coletas para 2 distintas espécies: $C$. papaya e $P$. niruri.

Dos 19 textos com locais de coleta "não identificados", 8 foram de C. papaya, destes, 5 são publicações de resumos apenas. E, 10 foram de $P$. niruri, sendo 7 resumos. Essas 2 espécies contêm cada uma 3 textos "não identificados" distintos de resumos. Outro texto "não identificado" é da espécie J. elliptica, sendo 1 resumo.

\section{LOCAIS DE ESTUDO}

Os locais de estudo, estão bem distribuídos pelo mundo (Figura 3). 


\section{DISTRIBUIÇÃO GEOGRÁFICA DAS COLETAS, ESTUDOS E PUBLICAÇÕES ACADÊMICAS SOBRE NOVE PLANTAS MEDICINAIS UTILIZADAS PARA O CONTROLE DE DIABETES EM MATO GROSSO}
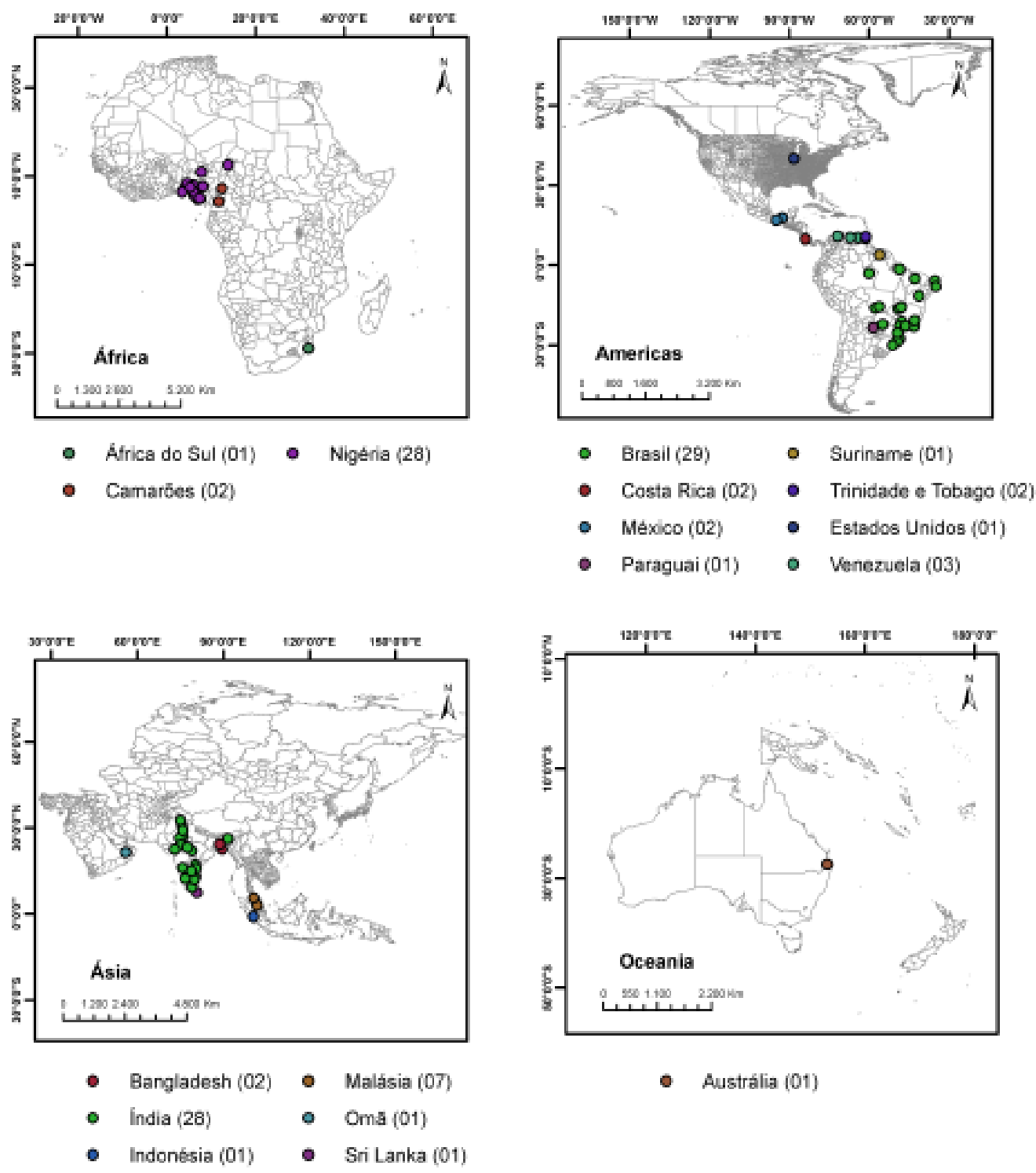

- Austrália (01)

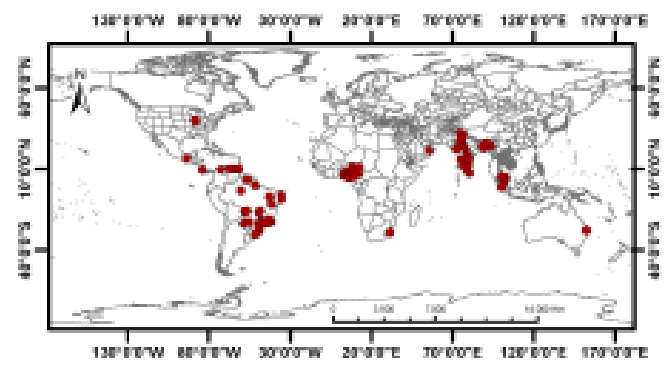

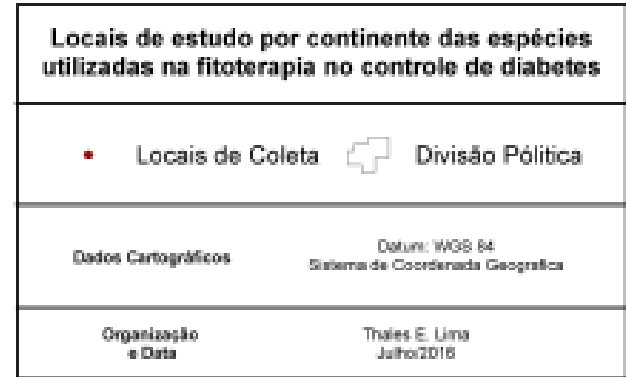

Figura 03 - Distribuição geográfica dos locais de estudo das publicações aproveitadas que se referem a fitoterapia no controle de diabetes envolvendo sete espécies utilizadas em Mato Grosso (MT), BRASIL (BR). Fonte de dados: Recuperado na Internet via Google acadêmico. Acessado em: Cáceres, MT, BR, entre 11 jul. 2013 à 30 jan. 2014. Organização: os autores. 


\section{DISTRIBUIÇÃO GEOGRÁFICA DAS COLETAS, ESTUDOS E PUBLICAÇÕES ACADÊMICAS SOBRE NOVE PLANTAS MEDICINAIS UTILIZADAS PARA O CONTROLE DE DIABETES EM MATO GROSSO}

Houve um aumento de textos aproveitados em que não se conseguiu identificar (23) seus locais de estudos, nem mesmo os continentes, comparando com os locais de coleta. Há uma distribuição muito diversa dos "locais identificados" (113) em que se realizaram os estudos. Porém se sobressai o continente americano $(41=36,28 \%)$, seguido do asiático $(40=35,39 \%)$ e africano $(31=27,43 \%)$. Dentro do continente americano, a América do Sul se destacou (34=30,08\%), confirmando a mesma ordem daqueles de locais de coleta. Conforme Calixto (2005, p.132), as publicações sobre plantas medicinais em 9 países da América Latina estão distribuídas entre Brasil 3722 (41,6\%), México 1781 (19,9\%), Argentina 1741 (19,5\%), Chile 573 (6,4\%), Venezuela 394 (4,4\%), Colômbia 265 (2,9\%), Peru 214 (2,4\%), Cuba 182 (2\%) e Uruguai 69 (0,8\%).

Os países "identificados" (113) com mais estudos foram Brasil (29=25,66\%), Índia e Nigéria, (28=24,77\% em ambas). Nesses 3 países, os estudos para espécies não apresentaram uma proporção similar (国 $=42,81 ; \alpha=1,1301 \mathrm{E}-08$ ). Segundo Motta et al. (2009, p.37) as pesquisas sobre o uso da medicina tradicional são mais frequentes em países pobres ou em desenvolvimento. Para C. papaya no Brasil, houve menos estudos que o esperado ( $\mathrm{Fo}=5$; $\mathrm{Fe}=15,35)$ e na Nigéria mais que o esperado (Fo=23; $F e=14,82$ ), enquanto para $P$. niruri, não houve diferença expressiva. Para as "demais espécies", o Brasil apresentou mais estudos do que o esperado ( $\mathrm{Fo}=17 ; \mathrm{Fe}=6,14)$. As espécies $C$. papaya e $P$. niruri abarcaram $78,82 \%$ do total de estudos destes 3 países (Brasil, Índia e Nigéria).

Entretanto, no Brasil, ainda são insuficiente os estudos sobre a utilização das plantas como medicamentos e sua inserção na cultura popular (VEIGA JUNIOR, 2008, p.308).

Contudo, através do Programa de Pesquisa de Plantas Medicinais, iniciado em 1983, o Brasil, exerceu uma ação multiplicadora, originando novos conhecimentos e a formação de recursos humanos de nível superior. Estes estudos geraram bases científicas para a utilização de plantas medicinais, como um recurso terapêutico. Mas, a interrupção do programa em 1995, foi negativo no desenvolvimento dessa área (SIMÕES, SCHENKEL, 2002, p.36). Apesar disto e do insuficiente investimento financeiro para pesquisa, tecnologia e inovação, a comunidade cientifica brasileira vem investigando, com limitações, o potencial das plantas. Mais recentemente, perspectivas animadores voltam a surgir com a Política Nacional de Plantas Medicinais e Fitoterápicos (BRASIL, 2009, p.20).

Há uma grande diversidade de estados (61) e cidades (71) em que foram realizados os estudos para as 7 espécies (Figura 3). Dentre as regiões identificadas do Brasil (29), há mais estudos no sudeste $(9=31,03 \%)$ e sul $(7=24,13 \%)$. Essa distribuição dos estudos é coerente com a abordagem de Guimarães (2006, p.07). O mesmo mostra que a geografia dos grupos com atividades de pesquisa em geral e de pesquisa em saúde, está distribuída assimetricamente entre as regiões brasileiras: sudeste (63\%), sul (17\%), nordeste $(13 \%)$, centro-oeste $(5 \%)$ e norte $(2 \%)$.

As proporções de textos aproveitados, para local de estudo das espécies, variaram entre os continentes ( $\chi 2=38,78 ; \mathrm{GL}=6 ; \alpha=7,8914 \mathrm{E}-07)$. As diferenças maiores estão para a espécie $C$. papaya, pois no continente americano esperavase haver 21 estudos, mas, constataram-se 10 e no africano esperava-se 16 e ocorreram 24, diante da hipótese de nulidade. Para $P$. niruri, não houve grande diferença. Para as demais espécies, esperava-se 6 no continente americano e teve 17 e no asiático esperava-se 6 estudos e teve 1 . As diferenças entre continentes na distribuição de frequências observadas (Fo) e esperadas (Fe) nos locais de estudo seguem a mesma tendência dos locais de coleta, ou seja, as coletas e os estudos para C. papaya ocorreram mais do que 0 esperado no continente africano e menos no americano. Os estudos das 7 espécies estão distribuídos entre 18 países (Figura 4).

Com relação a coletas "não identificadas" (19), houve um aumento de estudos "não identificados" (23), distribuídos 


\section{DISTRIBUIÇÃO GEOGRÁFICA DAS COLETAS, ESTUDOS E PUBLICAÇÕES ACADÊMICAS SOBRE NOVE PLANTAS MEDICINAIS UTILIZADAS PARA O CONTROLE DE DIABETES EM MATO GROSSO}

entre as espécies $C$. papaya (9), J. elliptica (1) e $P$.

niruri (13).

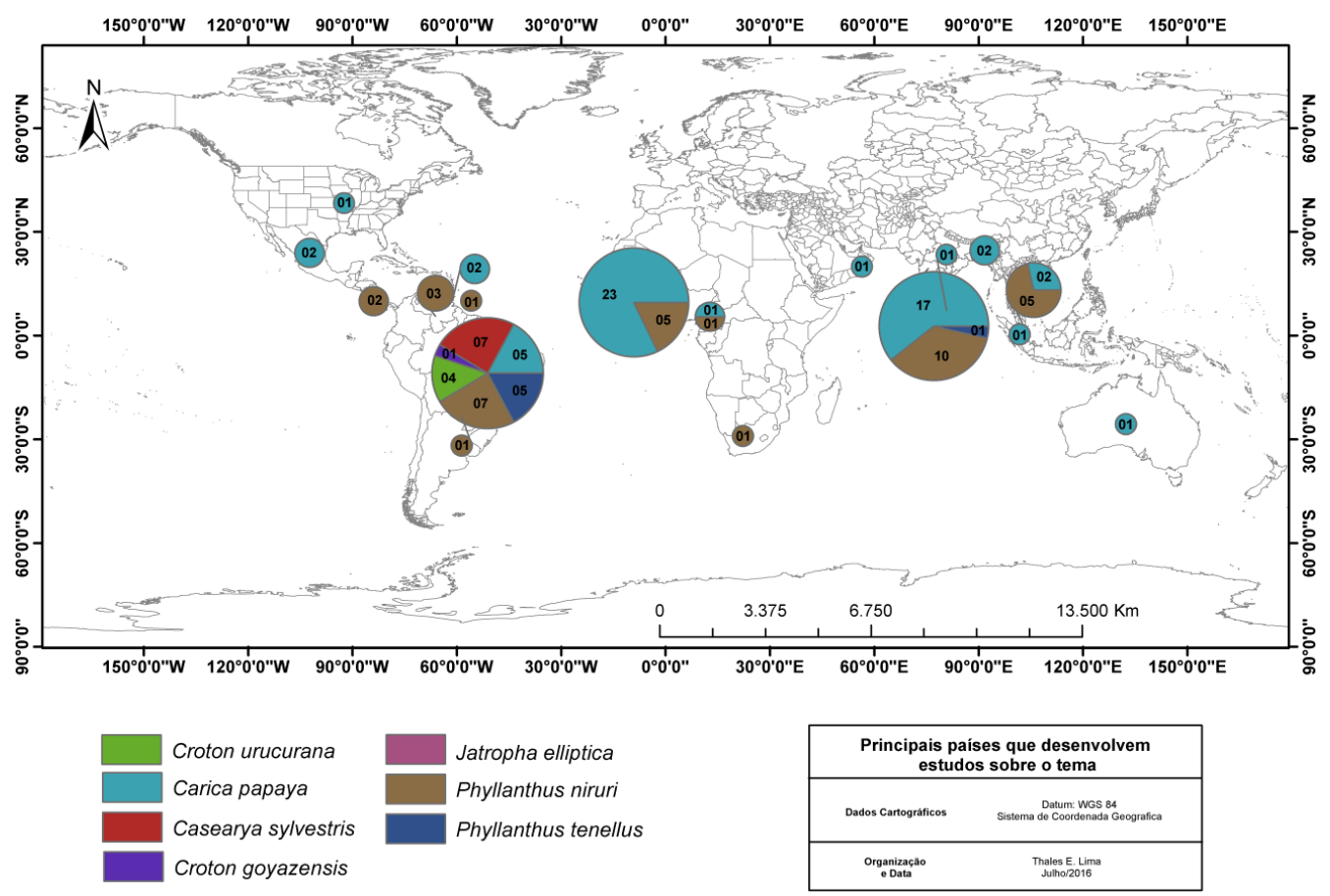

Figura 04 - Espécies e lugar geopolítico de estudo correspondente aos textos aproveitados que se referem a fitoterapia ao controle de diabetes envolvendo sete espécies utilizadas em Mato Grosso (MT), BRASIL (BR).Fonte de dados: Recuperados da Internet via Google acadêmico. Acessado em: Cáceres, MT, BR, entre 11 jul. 2013 à 30 jan. 2014. Organização: os autores.

Comparativamente, as coletas "não identificadas" (19), foram em menor número que os estudos "não identificados" (23); estes distribuídos entre as espécies C. papaya (9), J. elliptica (1) e $P$. niruri (13).

Os países com mais "estudos identificados" (113) foram Brasil (29=25,66\%), Índia e Nigéria com $28(24,77 \%$ x 2) textos cada uma. Os 2 países que concentraram mais estudos sobre C. papaya (59) e $P$. niruri (36), foram Nigéria $(23=38,98 \%)$ e Índia $(17=28,81 \%)$ para a primeira e, Índia (10=27,77\%) e Brasil $(7=19,44 \%)$ para a segunda espécie. As demais características da distribuição do estudo se assemelham a distribuição das coletas (Figura 2).

\section{LOCAIS DE PUBLICAÇÃO}

Os locais de publicação das espécies visadas estão apresentados em seu todo na figura 5 .
Em 37 (27,20\%) textos não se conseguiu identificar os locais de publicação. Para os casos identificados (99), predominaram publicações no continente americano ( $46=46,46 \%)$, seguido pelo asiático $(28=28,28 \%)$ e europeu $(13=13,13 \%)$. No americano, a América do Sul se destacou $(30=30,30 \%)$. Os países com mais publicações são Brasil $(25=25,25 \%)$ seguido da Índia $(23=23,23 \%)$ e Estados Unidos $(10=10,10 \%)$. A figura 5 mostra a distribuição das publicações nos demais países e continentes e a diversidade dos estados (50) e cidades (62).

Na América do Sul, o Brasil é o país com mais publicações resultantes de pesquisas sobre plantas medicinais no período de 1984-2004 (CALIXTO, 2005, p.132). No Brasil (25), os estados com maior número de publicações constantes na figura 5, são: São Paulo (9=36\%); seguido do Rio de Janeiro e Rio Grande do Sul com 3 (12\%) publicações cada; Distrito Federal, Minas Gerais e 


\section{DISTRIBUIÇÃO GEOGRÁFICA DAS COLETAS, ESTUDOS E PUBLICAÇÕES ACADÊMICAS SOBRE NOVE PLANTAS MEDICINAIS UTILIZADAS PARA O CONTROLE DE DIABETES EM MATO GROSSO}

Paraná com 2 (8\%) publicações cada e; os demais estados, com 1 trabalho.

As poucas publicações em alguns estados brasileiros pode sem atribuídas, em parte, a estruturação regional mais recente de Universidades, Instituições de pesquisa e de fomento desta. Segundo Guarim Neto e Morais (2003, p.563) as pesquisas com plantas medicinais em Mato Grosso, se evidenciam na década de 1980. Embora haja registros de estudos em expedições realizadas há mais de um século (RIEDER, GUARIM NETO, 2012, p.23).

Mas considerando estudos que não focam apenas uma determinada enfermidade, uma revisão bibliográfica efetuada no início deste século XXI, encontrou 101 trabalhos que abordam plantas medicinais do cerrado matogrossense (RIEDER, GUARIM NETO, 2012, p.24). 


\section{DISTRIBUIÇÃO GEOGRÁFICA DAS COLETAS, ESTUDOS E PUBLICAÇÕES ACADÊMICAS SOBRE NOVE PLANTAS MEDICINAIS UTILIZADAS PARA O CONTROLE DE DIABETES EM MATO GROSSO}
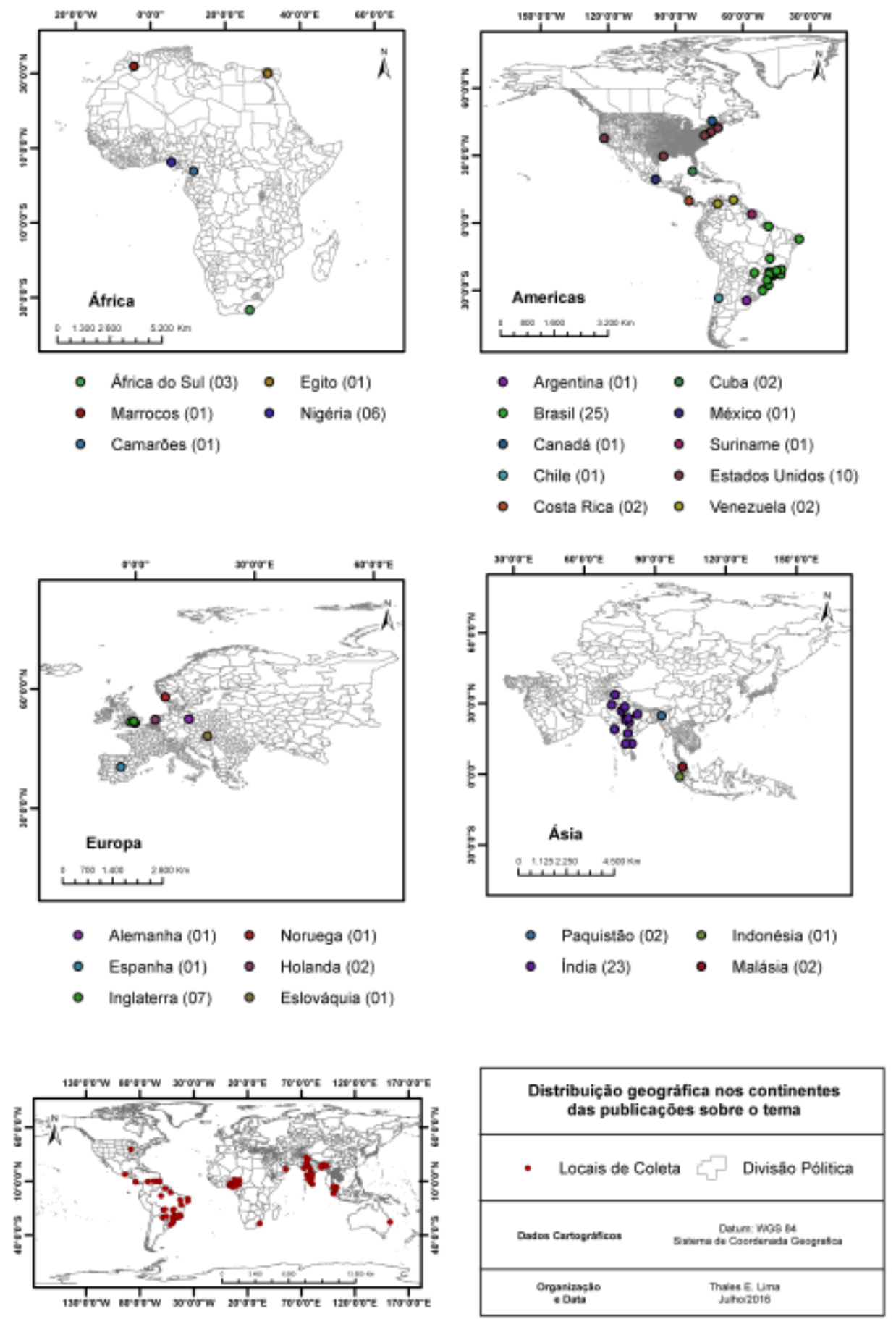

Figura 05 - Distribuição geográfica dos locais identificados de publicação dos textos aproveitados que se referem a fitoterapia no controle de diabetes envolvendo sete espécies utilizadas em Mato Grosso (MT), BRASIL (BR).Fonte de dados: Recuperado da Internet via Google acadêmico. Acessado em: Cáceres, MT, $B R$, entre 11 jul. 2013 à 30 jan. 2014. Organização: os autores.

A região sudeste do Brasil totaliza 14 (56\%) textos publicados e a região sul com 6
(24\%). Esta ordem de distribuição de publicações difere em parte do trabalho de Da Silva et al. 


\section{DISTRIBUIÇÃO GEOGRÁFICA DAS COLETAS, ESTUDOS E PUBLICAÇõES ACADÊMICAS SOBRE NOVE PLANTAS MEDICINAIS UTILIZADAS PARA O CONTROLE DE DIABETES EM MATO GROSSO}

(2013b, p.23). Eles verificaram a produção científica sobre DM tipo 2, disponibilizada no banco de teses e dissertações da Enfermagem da Coordenação de Aperfeiçoamento de Pessoal Nível Superior (CAPES), com base nos documentos selecionados, que a região brasileira com maior destaque foi a sudeste $(29=52,8 \%)$, seguida pelo nordeste $(13=23,6 \%)$, sul $(10=18,2 \%)$ e, em menor participação, as regiões centro-oeste $(2=3,6 \%)$ e norte $(1=1,8 \%)$. Nesta, o estado com maior número de publicações relacionados ao DM tipo 2 foi São Paulo (22=40\%) e o menor, Acre $(1=1,81 \%)$.

As proporções de textos aproveitados, para local de publicações das espécies, variaram entre os continentes $\left(\chi^{2}=22,94 ; \mathrm{GL}=6 ; \alpha=0,0008\right)$.

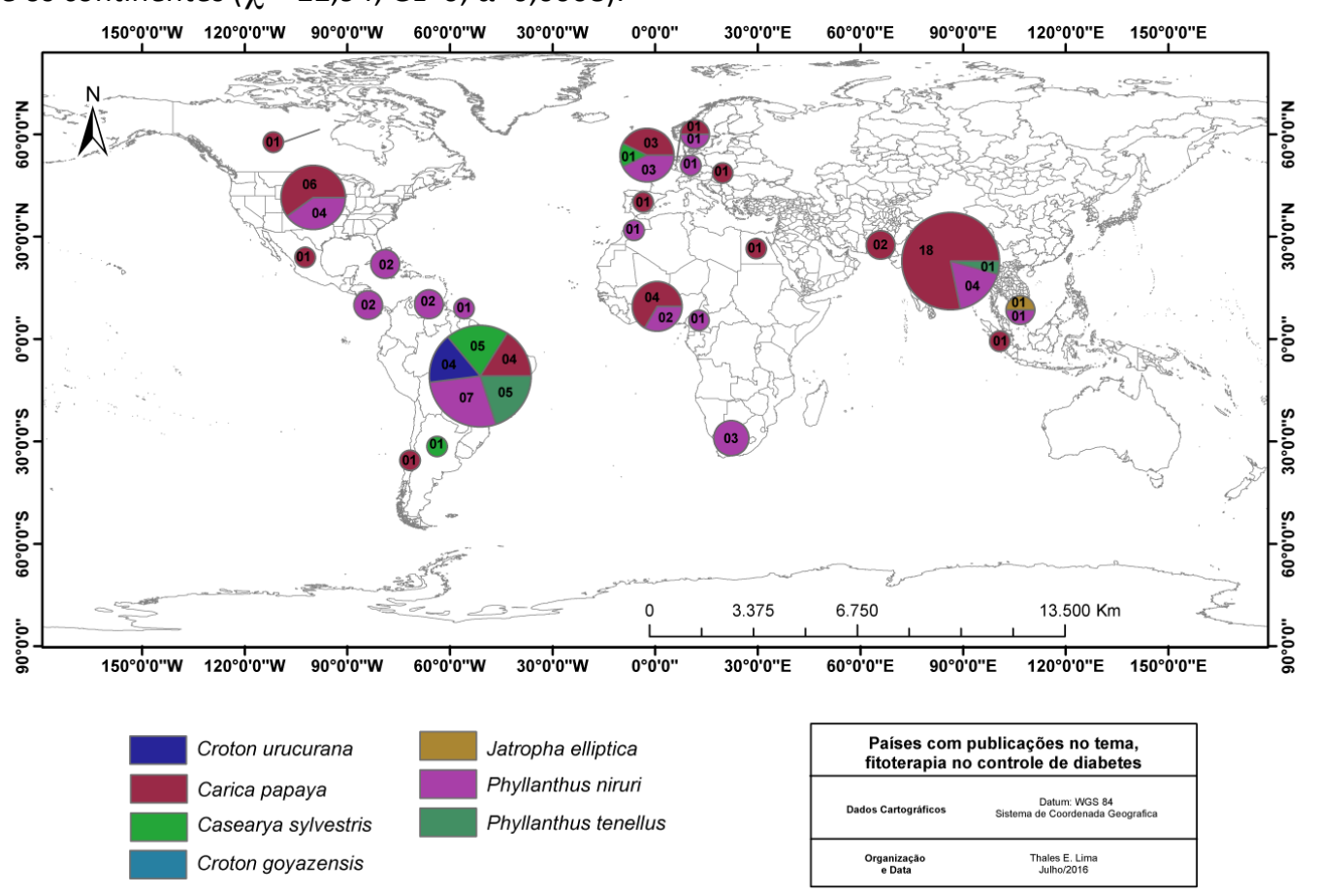

Figura 06 - Espécies e lugar geopolítico de publicação dos textos aproveitados que se referem a fitoterapia ao controle de diabetes envolvendo sete espécies utilizadas em Mato Grosso (MT), BRASIL (BR). Fonte de dados: Recuperados da Internet via Google acadêmico. Acessado em: Cáceres, MT, BR, entre 11 jul. 2013 à 30 jan. 2014. Organização: os autores.

Dos 136 textos aproveitados, em 37 não foi possível identificar o local de publicação. Entretanto, os textos com identificação de local de publicação (99) estão distribuídos entre 25 países. As publicações dos estudos sobre as espécies $C$. papaya (46) e $P$. niruri (35) se deram em 15 países cada uma. A Índia (18=39,13\%) e
As diferenças maiores estão para C. papaya, que no continente americano, diante da hipótese de nulidade, esperava-se 21 publicações, e encontraram-se 13, enquanto no asiático esperava-se 13 e ocorreram 21. Para $P$. niruri, não houve grandes diferenças. Para as "demais espécies", esperava-se 8 no continente americano e obteve-se 15. A distribuição entre os continentes das coletas, estudos e publicações para $P$. niruri apresentou-se dentro do esperado. 


\section{DISTRIBUIÇÃO GEOGRÁFICA DAS COLETAS, ESTUDOS E PUBLICAÇÕES ACADÊMICAS SOBRE NOVE PLANTAS MEDICINAIS UTILIZADAS PARA O CONTROLE DE DIABETES EM MATO GROSSO}

países da Europa $(13=13,13 \%)$ e nos Estados Unidos $(10=10,10 \%)$. Em alguns textos, para identificar o seu local de publicação, teve-se que entrar nos sites das revistas (periódicos) em que haviam sido publicados para assim encontrar suas cidades, e consequentemente estados e países. Mesmo assim, a proporção de local de publicação "não identificado" foi maior que os de estudos e coletas, conforme evidenciado na frase seguinte.

As proporções dos textos aproveitados, com locais "identificados" (li) e "não identificados" (ni), na fase de coleta ( $\mathrm{ni}=16,23 \%$; $\chi 2=3,16 ; \quad G L=2 ; \quad \alpha=0,2056)$, de estudos ( $\mathrm{ni}=20,35 \%$; 2 2=5,71; GL=2; $\alpha=0,0574$ ) e de suas publicações $\quad(n i=37,37 \% ; \quad \chi 2=5,57 ; \quad G L=2$; $\alpha=0,0615)$ mostraram ser independentes das espécies incluídas nesse trabalho, conforme a hipótese de nulidade. Portanto, as limitações para identificação do local de publicação é generalizado para as espécies. As instituições publicadoras podem solucionar esta questão.

Dados do Observatoire des Sciences et dês Techniques (OST) demonstram uma forte concentração na produção científica mundial, em 3 blocos de países: Europa (42,1\%), América do Norte (31,8\%) e Ásia (18,2\%), enquanto o Brasil participa com 1,1\%, apesar de esforços e progressos recentes (CONTINI, SÉCHET, 2011, p.30), o que contrasta em parte ao tratar-se de produção científica sobre plantas medicinais.

\section{CONSIDERAÇÕES FINAIS}

As coletas, estudos e publicações, dos textos aproveitados (136) concentram-se mais nas espécies $C$. papaya e $P$. niruri, representando $86,02 \%$ do total. As coletas, com locais "identificados" para as 7 espécies com textos aproveitados, distribuem-se diferentemente entre 4 continentes, 18 países e 75 cidades. Os estudos, com locais identificados de sua realização, estão distribuídos entre 4 continentes, 18 países e 71 cidades. Os estudos distribuíramse mais no Brasil, Índia e Nigéria, mas em proporções distintas.

As publicações, com locais "identificados", estão distribuídas distintamente entre 4 continentes, 25 países e 62 cidades. As publicações consideravelmente migram dos países de estudo para países desenvolvidos, no caso para Europa e América do Norte. As publicações, se concentraram mais em menos cidades que as coletas, mas estão dispersas em mais países.

\section{REFERÊNCIAS}

BADKE, M. R. Conhecimento popular sobre o uso de plantas medicinais e o cuidado de enfermagem, 2008. Tese (Mestrado em Enfermagem) - Programa de pós-graduação em enfermagem, Universidade Federal de Santa Maria, Santa Maria, 2008. Documento eletrônico. Disponível em: http://coral.ufsm.br/ppgenf/images/Mestrado/Di ssertacoes/2008_2009/MARCIO_ROSSATO_BADK E.pdf Acesso em: 08/04/2015.

BRASIL. Ministério da Saúde. Agencia Nacional de Vigilância Sanitária. Resolução da Diretoria Colegiada - RDC no 26, de 13 de maio de 2014. Diário Oficial da União. Brasília, 2014. Disponível em:

http://bvsms.saude.gov.br/bvs/saudelegis/anvisa /2014/rdc0026_13_05_2014.pdf Acesso em: 01/07/2016.

BRASIL. Ministério da Saúde. Secretaria de Atenção à Saúde. Departamento de Atenção Básica. Diabetes Mellitus / Ministério da Saúde, Secretaria de Atenção à Saúde, Departamento de Atenção Básica. - Brasília: Ministério da Saúde, 2006. 64 p. il. - (Cadernos de Atenção Básica, n. 16) (Série A. Normas e Manuais Técnicos). Disponível em: http://bvsms.saude.gov.br/bvs/publicacoes/diab etes_mellitus.PDF Acesso em: 29/04/2015.

BRASIL. Ministério da Saúde. Secretaria de Ciência, Tecnologia e Insumos Estratégicos. Departamento de Assistência Farmacêutica e Insumos Estratégicos. Programa Nacional de Plantas Medicinais e Fitoterápicos / Ministério da Saúde, Secretaria de Ciência, Tecnologia e Insumos Estratégicos, Departamento de Assistência Farmacêutica e Insumos Estratégicos. - Brasília: Ministério da Saúde, 2009. 136 p.: il. (Série C. Projetos, Programas e Relatórios). Disponível em: http://bvsms.saude.gov.br/bvs/publicacoes/prog rama_nacional_plantas_medicinais_fitoterapicos. pdf Acesso em: 28/04/2015. 


\section{DISTRIBUIÇÃO GEOGRÁFICA DAS COLETAS, ESTUDOS E PUBLICAÇÕES ACADÊMICAS SOBRE NOVE PLANTAS MEDICINAIS UTILIZADAS PARA O CONTROLE DE DIABETES EM MATO GROSSO}

BRASIL. Ministério da Saúde. Secretaria de Atenção à Saúde. Departamento de Atenção Básica. Práticas integrativas e complementares: plantas medicinais e fitoterapia na Atenção Básica/Ministério da Saúde. Secretaria de Atenção à Saúde. Departamento de Atenção Básica. - Brasília: Ministério da Saúde, 2012. 156 p.: il. - (Série A. Normas e Manuais Técnicos) (Cadernos de Atenção Básica; n. 31). Disponível em:

http://bvsms.saude.gov.br/bvs/publicacoes/prati cas_integrativas_complementares_plantas_medi cinais_cab31.pdf Acesso em: 28/04/2015.

CALIXTO, J. B. Efficacy, safety, quality control, marketing and regulatory guidelines for herbal medicines (phytotherapeutic agents). Brazilian Journal of Medical and Biological Research, v. 33, n.2, p. 179-189, 2000. Disponível em: http://www.scielo.br/scielo.php?pid=S0100$879 \times 2000000200004 \&$ script $=$ sci_arttext Acesso em: 08/04/2015.

CALIXTO, J. B. Twenty-five years of research on medicinal plants in Latin America: a personal view. Journal of ethnopharmacology, v. 100, n. 1, p. 131-134, 2005. Disponível em: http://medicinalherbs.comule.com/admin/article s/5098dc258ede0_sobia22.pdf Acessado em: 04/05/2015.

CONTINI, E.; SÉCHET, P. Ainda há um longo caminho para a ciência e tecnologia no Brasil. Revista Brasileira de Pós-Graduação, v. 2, n. 3, p. 30-39, 2011. Disponível em: http://ojs.rbpg.capes.gov.br/index.php/rbpg/arti cle/view/57/54 Acesso em: 08/04/2015.

DANTAS, J. L. L.; SOUZA, J, S.; PINTO, R. M. S.; LIMA, J. F. Variabilidade genética e melhoramento do mamoeiro. Recursos Genéticos e Melhoramento de Plantas para o Nordeste Brasileiro (online). Versão, v. 1, 1999. Disponível em:

http://www.ceinfo.cnpat.embrapa.br/arquivos/a rtigo_2567.pdf Acesso em: 09/04/2015.

DA SILVA, S. C. S.; MAMEDE, M. E.; VIANA, E. S.; DRUZIAN, J. I. Mapeamento tecnológico para projeto de pesquisa de mamão desidratado com adição de frutooligossacarídeo entre 1983 e 2012. Anais do Simpósio Internacional de Inovação Tecnológica-SIMTEC, v. 1, n. 1, p. 796809, 2013. (A) Disponível em: http://www.portalmites.com.br/anaissimtec/ind ex.php/simtec/article/view/81/96 Acesso em: 08/04/2015.
DA SILVA, M. M.; BUDÓ, M. L. D.; SIMON, B. S.; ROSSO, L. F. Tendência da produção científica sobre diabetes mellitus nas teses e dissertações da enfermagem brasileira. Saúde (Santa Maria), v. 39, n. 1, p. 21-31, 2013. (B) Disponível em: http://periodicos.ufsm.br/revistasaude/article/vi ew/7613/pdf Acesso em: 08/04/2015.

Diretrizes da Sociedade Brasileira de Diabetes (SBD): 2013-2014/Sociedade Brasileira de Diabetes; [organização José Egidio Paulo de Oliveira, Sérgio Vencio]. - São Paulo: AC Farmacêutica, 2014. Disponível em: http://www.sgc.goias.gov.br/upload/arquivos/20 14-05/diretrizes-sbd-2014.pdf Acesso em: 02/05/2015.

GUARIM NETO, G.; MORAIS, R. G. Recursos medicinais de espécies do cerrado de Mato Grosso: um estudo bibliográfico. Acta Botanica Brasílica, v. 17, n. 4, p. 561-584, 2003. Disponível em:

http://www.scielo.br/pdf/abb/v17n4/a09v17n4 Acesso em: 09/04/2015.

GUIMARÃES, J. A. A pesquisa médica e biomédica no Brasil: comparações com o desempenho científico brasileiro e mundial. Ciências e saúde coletiva, v. 9, n. 2, p. 303-327, 2004. Disponível em:

http://www.scielo.br/pdf/\%0D/csc/v9n2/20387.p df Acesso em: 09/04/2015.

GUIMARÃES, R. Pesquisa em saúde no Brasil: contexto e desafios. Revista de Saúde Pública, v. 40, n. Esp, p. 3-10, 2006. Disponível em: http://www.scielosp.org/pdf/rsp/v40nspe/30616 .pdf? Acesso em: 25/03/2015.

MACIEL, M. A. M.; PINTO, A. C.; VEIGA JUNIOR, V.F. Plantas medicinais: a necessidade de estudos multidisciplinares. Química nova, v. 25, n. 3, p. 429-438, 2002. Disponível em: http://www.scielo.br/pdf/\%0D/qn/v25n3/9337.p df Acesso em: 24/03/2015.

MEDEIROS, B. J. L. Estudo pré-clínico do Extrato Hidroetanólico de Calophyllum brasiliense Cambess.: atividades hipoglicemiante e toxidade, 2014. Tese (Mestrado em Ciências da Saúde) Departamento de Pesquisa e pós-graduação em Ciências da Saúde, Universidade Federal do Amapá, Amapá, 2014. Documento eletrônico. Disponível em: http://www2.unifap.br/ppcs/files/2014/09/Bene dito-Junior-Lima-de-Medeiros.pdf Acesso em: 26/03/2015. 


\section{DISTRIBUIÇÃO GEOGRÁFICA DAS COLETAS, ESTUDOS E PUBLICAÇÕES ACADÊMICAS SOBRE NOVE PLANTAS MEDICINAIS UTILIZADAS PARA O CONTROLE DE DIABETES EM MATO GROSSO}

MOTTA, J. M. C.; BARROS, N. F.; CASTELLANOS, M. E. P.; ALEGRE, S. M.; TOVEY, P.; BROOM, A. Medicina alternativa e complementar e diabetes mellitus: uma articulação a ser estudada. Brasília Médica, vol. 1, n. 46, p. 36-45, 2009. Disponível em:

https://repositorio.ufba.br/ri/bitstream/ri/2040/ 1/per\%20nac2009.2.pdf Acesso em: 25/03/2015.

NEGRI, G. Diabetes melito: plantas e princípios ativos naturais hipoglicemiantes. Brazilian Journal of Pharmaceutical Sciences, v. 41, n. 2, p. 121142, 2005. Disponível em: http://www.scielo.br/pdf/\%0D/rbcf/v41n2/2803 4.pdf Acesso em: 24/03/2015.

PEREIRA, S. C. G. Plantas medicinais e medicamentos à base de plantas na terapêutica da diabetes, 2012. Tese (Mestrado integrado em Ciências Farmacêuticas) - Faculdade de Farmácia, Universidade de Lisboa, Lisboa, 2012. Documento eletrônico. Disponível em: http://www.portaldoconhecimento.gov.cv/bitstr eam/10961/2578/1/MONOGRAFIA.pdf Acesso em: 25/03/2015.

REZENDE, H.A.; COCCO, M.I.M. A utilização de fitoterapia no cotidiano de uma população rural. Revista da Escola de Enfermagem da USP, v. 3, n. 36 p. 282-288, 2002. Disponível em: http://www.scielo.br/pdf/reeusp/v36n3/v36n3a1 0 Acesso em: 22/03/2015.

RIEDER, A.; GUARIM NETO, G. Saúde e Ambiente: Plantas medicinais utilizadas para controle de diabetes em Mato Grosso, Brasil. Cáceres: UNEMAT Editora, 2012.

RIEDER, A.; RODRIGUES, F. A. C. Recuperação e filtragem de textos sobre saúde e ambiente através de mecanismos de busca na internet. Cáceres: UNEMAT, p. 1-25, 2012. (Artigo submetido à revista Informação \& Sociedade em 2014).

SIMÕES, C. M. O.; SCHENKEL, E. P. A pesquisa e a produção brasileira de medicamentos a partir de plantas medicinais: a necessária interação da indústria com a academia. Revista brasileira de farmacognosia, v. 12, n. 1, p. 35-40, 2002. Disponível em: http://www.scielo.br/pdf/rbfar/v12n1/a05v12n1. pdf Acesso em: 26/03/2015.

VEIGA JUNIOR, V. F. Estudo do consumo de plantas medicinais na Região Centro-Norte do Estado do Rio de Janeiro: aceitação pelos profissionais de saúde e modo de uso pela população. Revista brasileira de farmacognosia, v. 18 , n. 2, p. 308-13, 2008. Disponível em: https://www.researchgate.net/profile/Valdir_Vei ga/publication/262496470_Study_of_the_medici nal_plants_consumption_in_the_Middle-

North_Region_of_the_Rio_de_Janeiro_State_acc eptance_by_health_professionals_way_of_use_o f_the_population/links/54b29d490cf28ebe92e2c f04.pdf Acesso em: 25/03/2015.

VOLPATO, G. T.; DAMASCENO, D. C.; CALDERON, I. M. P.; RUDGE, M. V. C. Revisão de plantas brasileiras com comprovado efeito hipoglicemiante no controle do Diabetes mellitus. Revista Brasileira de Plantas Medicinais, v. 4, n. 2, p. 35-45, 2002. Disponível em: http://repositorio.unesp.br/bitstream/handle/11 449/66787/2-s2.00036087777. pdf?sequence $=1$ \&isAllowed $=y$ Acesso em: 23/03/2015 